\title{
Analytical Framework for End-to-End Delay Based on Unidirectional Highway Scenario
}

\author{
Aslinda Hassan, ${ }^{1}$ Mohamed H. Ahmed, ${ }^{2}$ and M. A. Rahman ${ }^{2}$ \\ ${ }^{1}$ Faculty of Information and Communication Technology, Universiti Teknikal Malaysia Melaka, Hang Tuah Jaya, \\ 76100 Durian Tunggal, Melaka, Malaysia \\ ${ }^{2}$ Faculty of Engineering and Applied Science, Memorial University of Newfoundland, St. John's, NL, Canada A1B 3X5
}

Correspondence should be addressed to Aslinda Hassan; aslindahassan@utem.edu.my

Received 26 September 2014; Revised 15 April 2015; Accepted 18 May 2015

Academic Editor: Francisco Chicano

Copyright (C) 2015 Aslinda Hassan et al. This is an open access article distributed under the Creative Commons Attribution License, which permits unrestricted use, distribution, and reproduction in any medium, provided the original work is properly cited.

In a sparse vehicular ad hoc network, a vehicle normally employs a carry and forward approach, where it holds the message it wants to transmit until the vehicle meets other vehicles or roadside units. A number of analyses in the literature have been done to investigate the time delay when packets are being carried by vehicles on both unidirectional and bidirectional highways. However, these analyses are focusing on the delay between either two disconnected vehicles or two disconnected vehicle clusters. Furthermore, majority of the analyses only concentrate on the expected value of the end-to-end delay when the carry and forward approach is used. Using regression analysis, we establish the distribution model for the time delay between two disconnected vehicle clusters as an exponential distribution. Consequently, a distribution is newly derived to represent the number of clusters on a highway using a vehicular traffic model. From there, we are able to formulate end-to-end delay model which extends the time delay model for two disconnected vehicle clusters to multiple disconnected clusters on a unidirectional highway. The analytical results obtained from the analytical model are then validated through simulation results.

\section{Introduction}

In general, a vehicular ad hoc network (VANET) is formed between nodes on as-needed basis. To create a VANET, vehicles need to have wireless transceivers and computerized modules that enable the vehicles to act as network nodes. Vehicle-to-vehicle and vehicle-to-infrastructure communications using wireless short-range technologies allow vehicles to broadcast and share information that can be used in different types of applications such as active road safety applications, traffic efficiency applications, and infotainment applications [1]. In a VANET, when the distance between two vehicles is less than the communication range of the vehicles, these vehicles are able to communicate with each other using the wireless channel. Nevertheless, in a sparse vehicular network, the distance between two vehicles is usually larger than the communication range. Therefore, in this type of network, a vehicle normally employs a carry and forward approach, where it holds the message it wants to transmit until the vehicle meets other vehicles or roadside units.
Consequently, the end-to-end delay in VANET is usually high. The study of the end-to-end delay in VANET can be considered as one of the most important investigations in vehicular network because extensive applications such as active safety and emergency response applications [2] require the messages to be transmitted with minimal delay.

Wu et al. [3] have determined an information propagation speed model in a VANET that is based on the carry and forward scheme. However, the derivation of the probability distribution for the time delay when using carry and forward approach or catch-up delay model is limited to only that between a cluster with informed vehicles and another cluster with uninformed vehicles, and the probability distribution of the catch-up model is not presented in the numerical results. Therefore, we are not able to ascertain the type of distribution for the catch-up model.

In this paper, we produce a closed form solution for the catch-up delay model from Wu et al. [3]. By producing a closed form solution for the catch-up time delay model between two disconnected clusters, we are able to 
approximate the distribution of the catch-up time delay model as an exponential distribution by using regression analysis which, to the extent of our knowledge, has not been done in the literature. Next, we investigate and derive a new distribution model to represent the number of disconnected vehicle clusters on a unidirectional highway. Using a Poisson process as our basic assumption for the arrival of vehicles and vehicle-to-vehicle (V2V) connectivity models, we formulate the distribution model for number of vehicle clusters in a certain length of a highway. Using this model, we are able to estimate the number of vehicle clusters in both sparse and dense networks. The analytical model for number of disconnected clusters is then utilized in our investigation to develop a distribution model for end-to-end delay on a highway for multiple disconnected vehicle clusters using V2V connectivity parameters that include the carry and forward mechanism. We are able to further determine the probability distribution model for the end-to-end delay between the multiple disconnected clusters as an Erlang $-n$ distribution by using the exponential approximation of the catch-up delay between two disconnected clusters and the models for the multiple number of clusters. This framework enables us to assess the end-to-end delivery delay between a source and destination moving on a highway for both disconnected and well-connected vehicular networks.

\section{Related Works}

Although many research studies have been carried out to incorporate the carry and forward approach in their proposed routing protocols, not many studies have been done in analyzing the end-to-end delay when the carry and forward approach is employed during the packet forwarding in VANET. Wu et al. [3] have presented an analytical study on the information propagation speed when the carry and forward approach is used in both one- and two-way highway scenarios where vehicle arrivals are based on Poisson process and the vehicle speeds are uniformly distributed in a designated range. The authors provide numerical results on information propagation speed under two network models, which are low density network and high density network.

A number of researches have developed analytical models for studying vehicular network characteristics and performance metrics [4-9]. However, these studies are focusing mainly on information propagation speed model, connectivity model, mobility model, and link reliability model. They do not present any probability distribution model on endto-end delay and the information propagation speed model is normally based on the expected values of the end-toend delivery delay. Zhang et al. in [6] have modified the information propagation speed model from $\mathrm{Wu}$ et al. [3] by using a traffic density for Poisson arrival model and truncated Gaussian distribution for vehicles' speed. Nevertheless, the study done by [6] does not show any distribution model for the catch-up delay. Furthermore, the authors only include the results on the expected value for the information propagation speed for a VANET highway. The study does not show any development on the distribution model for end-to-end delay.
Wisitpongphan et al. [10] proposed a similar analytical model as [3] for VANET connectivity in a sparse network. Using empirical traffic data, the authors study and formulate VANET parameters such as interarrival time and intervehicle spacing. The authors also derive a comprehensive analytical framework that can be used to characterize a sparse vehicular network for one- and two-directional highways. Furthermore, the authors did an analysis on a parameter similar to the catch-up time in [3] which is referred to as the rehealing time. However, the study on rehealing time in [10] is focused on the two-directional highways and between adjacent vehicles. Through simulation, the authors are able to validate their analytical framework and analyze end-to-end delay for packet transmission with distance between source and destination varying from 1 to $30 \mathrm{~km}$ [10]. Nonetheless, the study on the end-to-end delay in [10] is based on the expected values and purely based on simulation results. There is no distribution model derived for the end-to-end parameter.

A study on end-to-end delay model is done in [11] where the authors analyze the total delay time needed by a relay to carry a packet from a source to a destination using the carry and forward system. The main goal of this study is to find the relay's optimal location that minimizes the total delay while taking into account the effect of channel fading, path loss, and forward error correction. However, the study is based on a mobile ad hoc network scenario with only one relay between the source and destination.

In [12], the author also proposes a similar study on the end-to-end model, where the author uses the ergodic Markov chain to model the vehicle's mobility, the exponential distribution for the initial vehicle density, and the normal distribution for the average vehicle speed. The author creates the model for vehicles that are sparsely arranged on a onedirectional straight road. Using these assumptions, the author is able to obtain expressions for the exact delay time and delivery ratio. Nevertheless, the model in [12] only considered transmission between two vehicles, not between clusters of vehicles. In addition, the model does not consider the carry and forward approach during packet transmissions. Instead, the authors use $T$-seconds-wait rule where the packets are discarded if vehicles are unable to transmit them within $T$ seconds.

\section{System Model of the Time Duration for the Catch-Up Phase}

Consider a scenario presented in Figure 1 where a number of vehicles independently travel along a unidirectional highway of length $D$ meters. The speed of each vehicle, $V$, is modeled using uniform distribution over $\left[v_{\min }, v_{\max }\right]$. Based on the studies done in $[3,8,13]$, vehicles' speed can be represented with uniform distribution with interval $\left[v_{\min }, v_{\max }\right]$, where each vehicle freely moves at its chosen velocity. Each vehicle is then assumed to move along the highway at a constant speed, $v \mathrm{~m} / \mathrm{s}$, such that the distance between the vehicle and its neighbors remains unchanged. The model assumes that vehicles arrive to the highway following a Poisson process with an average rate equal to the traffic flow rate (vehicles per unit time). Empirical studies have shown that Poisson 


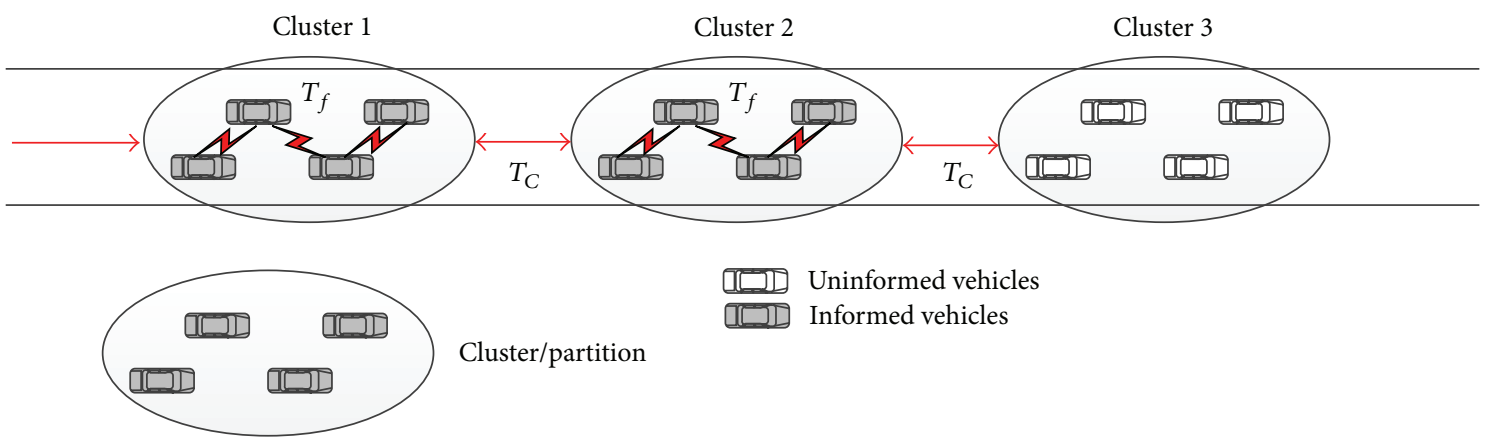

FIGURE 1: Example of message propagation scenario.

arrival model can be used to model vehicle arrival rate in free flow phase [10] and it is commonly used model in the studies of VANETs [6-9]. The vehicles are then partitioned into a number of clusters. In this model, a cluster can be defined as a group of vehicles that are able to propagate messages using multihop forwarding via wireless channel. Road traffic statistics in [14] have shown that vehicles tend to travel in clusters on a highway. The clusters, which are formed in the highway, are split and merged over time due to the mobility of the vehicles. If the gap between two clusters is larger than the transmission range, $r$, then the carry and forward strategy is used to forward messages. A vehicle is considered as an informed vehicle if the vehicle has the message that needs to be transmitted. In this scenario, we also assume that the source of the messages is located in a cluster of informed vehicles and the receiver of the messages is found in an uninformed cluster located at the end of the highway. The message is transmitted via one of two ways, through either the forward process or the catch-up process. In the forward process, the message is forwarded to other neighboring vehicles within a partition via the wireless channel, where the message rapidly propagates hop by hop until it reaches the farthest vehicle of that partition. In the catch-up process, the message travels along with the carrying vehicle until the carrying vehicle arrives within the communication range of the last uninformed vehicle in the partition ahead of it. Once the carrying vehicle is inside the partition with a group of uninformed vehicles, the message will be again propagated via forward process. Both processes alternate with each other as the message propagates along the road.

The term $T_{C}$ in Figure 1 is the time duration for the catchup process, where packets are being carried by the carrying vehicle until the vehicle is able to forward the packets via wireless transmission to the last uninformed vehicle in the partition ahead of it. Although the open form cumulative distribution function (CDF) of $T_{C}$ has been extensively studied and derived in [3], as shown in (1), the authors do not present the CDF or probability density function (PDF) of $T_{C}$ in their numerical results. Based on the assumptions, expressions, notations, and model parameters provided by the authors in [3], we are able to produce the CDF and PDF of $T_{C}$ via numerical integration. Notations and model parameters needed for the derivation of $T_{C}$ distribution are shown in Notations in this analysis.
The cumulative distribution function $(\mathrm{CDF})$ of $T_{C}$ as presented in [3] is shown in

$$
\begin{aligned}
& F_{T_{c}}(t) \\
& \quad=\int_{r}^{\infty}\left[\int_{r}^{\infty} F_{X^{\prime}(t)}(x+r-l) f_{X(t)}(x) d x\right] f_{L_{U C}}(l) d l .
\end{aligned}
$$

Based on (1), we conclude that we need to derive closed form solutions for $f(x ; t), F\left(x^{\prime}, t\right)$, and $f_{L_{U C}}(l)$, which are not presented in [3].

3.1. The Derivation of Closed Form Solution for CDF and PDF of Message Propagation Distance, $X(t)$. Let $X(t)$ denote the distance traveled by a first vehicle in the front most informed cluster after passing a random location, $H$, during the time interval $[0, t]$ (refer to Figure 1). The CDF of $X(t)$ is expressed as

$$
F(x, t)=\sum_{n=0}^{\infty} P[X(t)<x \mid N(t)=n] P[N(t)=n]
$$

where $P[X(t)<x \mid N(t)=n]$ is given as

$$
\begin{aligned}
& P[X(t)<x \mid N(t)=n] \\
& \quad=P\left[V_{0} t<x, V_{i}\left(t-T_{i}\right)<x \text { for each } i=1,2, \ldots, n\right] .
\end{aligned}
$$

Let $V_{0}$ in (3) denote the speed of the source vehicle located at the location $H$ at time, $t=0$. Let $V_{i}$ denote the speed of the vehicle $i$ and let $T_{i}$ denote the arrival time of a vehicle $i$ at location $H$ after $t=0$.

Based on the assumption of a Poisson arrival, $N(t)$ is defined as the number of vehicles arriving at the highway during $(0, t]$ and expressed as

$$
P[N(t)=n]=\frac{e^{\lambda t}(\lambda t)^{n}}{n !} .
$$

Using the Poisson process theorem $[15,16]$, given that $n$ vehicles have passed the location $H$ between time $(0, t]$, the arrival times $T_{1}, \ldots, T_{n}$ at which the events occur, considered as unordered random variables, are distributed independently and uniformly in the interval $(0, t)$. Therefore, with 
this theorem, $T_{i}$ can be presented as $T_{i} \sim \operatorname{uniform}(0, t)$. With the assumptions that $T_{1}, T_{2}, \ldots, T_{n}$ and $V_{1}, V_{2}, \ldots, V_{n}$ are independent and identically distributed (i.i.d.) and uniformly distributed at the intervals $(0, t]$ and $\left[v_{\min }, v_{\max }\right]$, respectively, (3) can be expressed as

$$
\begin{aligned}
P & {[X(t)<x \mid N(t)=n] } \\
& =P[V t<x] P[V(t-T)<x]^{n} .
\end{aligned}
$$

Let $V$ denote the vehicles' speed and it is uniformly distributed at interval $\left[v_{\min }, v_{\max }\right]$. Therefore, $P[V t<x]$ can be expressed as

$$
P[V t<x]= \begin{cases}0, & \frac{x}{t}<v_{\min } \\ \frac{(x / t)-v_{\min }}{v_{\max }-v_{\min }}, & v_{\min } \leq \frac{x}{t} \leq v_{\max } \\ 1, & \frac{x}{t}>v_{\max } .\end{cases}
$$

The probability $P[V(t-T)<x]$ in (5) denotes the probability of the distance traveled by $n$ vehicles after passing the location $H$ at the speed between the interval $\left[v_{\min }, v_{\max }\right]$ and at the time in the interval $(0, t)$. With the assumption that the random variable $T$ is independent and identically distributed (i.i.d.) and uniformly distributed in the interval $(0, t]$, the probability can be formulated as

$$
\begin{aligned}
P & {[V(t-T)<x] } \\
& =\int_{v_{\min }}^{v_{\max }} P\left[t-T \leq \frac{x}{V} \mid V=v\right] f_{V}(v) d v \\
& =\int_{v_{\min }}^{v_{\max }} P\left[T>t-\frac{x}{v}\right] f_{V}(v) d v . \\
& =\int_{v_{\min }}^{x / t} 1 \cdot f_{v}(v) d v+\int_{x / t}^{v_{\max }} \frac{x}{v t} \cdot f_{v}(v) d v, \\
P & {[V(t-T)<x]=\frac{x-t v_{\min }+x \ln \left(v_{\max } /(x / t)\right)}{t\left(v_{\max }-v_{\min }\right)} . }
\end{aligned}
$$

Using (4), (6), and (7), we are able to solve (2), which is the CDF of $X(t)$ and is shown in

$$
\begin{array}{r}
F(x, t)=\sum_{n=0}^{\infty} P[X(t) \mid N(t)=n] P[N(t)=n] \\
=\sum_{n=0}^{\infty} P[V t<x] P[V(t-T)<x]^{n} \frac{e^{\lambda t}(\lambda t)^{n}}{n !} \\
=\frac{(x / t)-v_{\min }}{v_{\max }-v_{\min }} \\
\cdot \sum_{n=0}^{\infty}\left(\left(\frac{x-t v_{\min }+x \ln \left(v_{\max } /(x / t)\right)}{t}\right)^{n}\right.
\end{array}
$$

$$
\begin{gathered}
\left.\cdot \frac{e^{-\lambda t}(\lambda t)^{n}}{n !}\right), \\
F(x, t)= \begin{cases}\frac{(x / t)-v_{\min }}{e^{\lambda t}\left(v_{\max }-v_{\min }\right)} \cdot e^{\sigma_{1}} ; & v_{\min } \leq \frac{x}{t} \leq v_{\max } \\
1 ; & \frac{x}{t}>v_{\max },\end{cases}
\end{gathered}
$$

where

$$
\sigma_{1}=\frac{\lambda\left(x-t v_{\min }+x \ln \left(t v_{\max } / x\right)\right)}{v_{\max }-v_{\min }} .
$$

Next, we take the derivative of $F_{X(t)}(x)$ to derive the PDF of $X(t)$, which is displayed in

$$
\begin{aligned}
f(x ; t)= & \frac{d F_{X(t)}}{d x} \\
= & \frac{e^{\sigma_{2}}}{t e^{\lambda t}\left(v_{\max }-v_{\min }\right)} \\
& -\frac{\lambda \sigma_{1} \ln \left(v_{\max } /(x / t)\right)\left(v_{\min }-x / t\right)}{e^{\lambda t}\left(v_{\max }-v_{\min }\right)^{2}},
\end{aligned}
$$

where

$$
\sigma_{2}=\frac{\lambda \sigma_{1}}{v_{\max }-v_{\min }} .
$$

3.2. The Derivation of Closed Form Solution for Cumulative Distribution Function of $X^{\prime}(t)$ : Distance that the Partition Tail Moves. Let $X^{\prime}(t)$ denote the distance traveled by the last vehicle in a uninformed cluster that is in front of an informed cluster during the time interval $[0, t]$. The CDF of $X^{\prime}(t)$ is expressed in

$$
\begin{aligned}
& F\left(x^{\prime}, t\right) \\
& =1 \\
& \quad-\sum_{n=0}^{\infty} P\left[X^{\prime}(t)>x \mid N^{\prime}(t)=n\right] P\left[N^{\prime}(t)=n\right],
\end{aligned}
$$

where

$$
\begin{aligned}
P & {\left[X^{\prime}(t)>x \mid N^{\prime}(t)=n\right] P\left[N^{\prime}(t)=n\right] } \\
& =P\left[V_{0} t>x, V_{i}\left(t-T_{i}\right) \text { for each } i=1,2, \ldots, n\right] .
\end{aligned}
$$

In (13), $V_{0}$ is defined as the speed of the uninformed vehicle at location $J$ at time $t=0$ and $N^{\prime}(t)$ denote the number of vehicles that pass location $J$. As shown in Figure 1, we conclude there are no other uninformed vehicles passing location $J$ after the last uninformed vehicle during a catch-up phrase, and hence the earliest time for the last uninformed vehicle to pass location $J$ is $-\left(v_{\max } / v_{\min }-1\right) t$, with the condition that the vehicle is in location $J$ at the time 0 and the vehicle speed is in the interval $\left[v_{\min }, v_{\max }\right]$. Using the same 
properties of independent and identically distributed (i.i.d) for $T_{i}$ and $V_{i}$ as in Section 3.1, (13) can be rewritten as

$$
\begin{gathered}
P\left[X^{\prime}(t)>x \mid N^{\prime}(t)=n\right] P\left[N^{\prime}(t)=n\right] \\
=P(V t>x) P[V(t-T)>x]^{n} .
\end{gathered}
$$

Next, given that $N(t)=n$, under the properties of the Poisson process [15], the $n$ jump epochs $T_{1}, \ldots, T_{n}$ have the same distribution as the order statistics corresponding to $n$ independent variables uniformly distributed on the interval $\left[-\left(v_{\max } / v_{\min }-1\right) t, 0\right]$. Therefore, (4) has to be rewritten according to the the new distribution of $T$, which is shown in

$$
\begin{aligned}
P[ & \left.N^{\prime}(t)=n\right] \\
& =\frac{e^{-\lambda\left(v_{\max } / v_{\min }-1\right) t}\left(\lambda\left(v_{\max } / v_{\min }-1\right) t\right)^{n}}{n !} .
\end{aligned}
$$

In (14), $P(V t>x)$ is expressed as

$$
\begin{aligned}
& P(V t>x)=1-P(V t<x), \\
& P(V t>x)=\frac{v_{\max }-x / t}{v_{\max }-v_{\min }}
\end{aligned}
$$

Let $P[V(t-T)>x]$ denote the probability of the distance traveled by $n$ vehicles after passing location $J$ in the interval of $\left[-\left(v_{\max } / v_{\min }-1\right) t, 0\right]$ with a speed between $\left[v_{\min }, v_{\max }\right]$. Therefore, (7) can be rewritten as

$$
\begin{aligned}
P[V(t-T)>x] & \\
= & \int_{v_{\min }}^{x / t} P\left[T<t-\frac{x}{v}\right] f_{V}(v) d v \\
& \quad+\int_{x / t}^{v_{\max }} P\left[T<t-\frac{x}{v}\right] f_{V}(v) d v \\
= & \frac{v_{\min }\left(x / t-v_{\min }\right)}{\left(v_{\max }-v_{\min }\right)^{2}} \\
& -\frac{x v_{\min }}{t v\left(v_{\max }-v_{\min }\right)^{2}}\left[\ln \left(\frac{x}{t}\right)-\ln \left(v_{\min }\right)\right]+1 .
\end{aligned}
$$

Finally, we substitute (15), (16), and (17) in (12) to solve $F\left(x^{\prime}, t\right)$, which is shown in

$$
\begin{aligned}
& F\left(x^{\prime}, t\right)=1-P[V t>x] \sum_{n=0}^{\infty}\left[P[V(t-T)>x]^{n}\right. \\
& \left.. \frac{e^{-\lambda\left(v_{\max } / v_{\min }-1\right) t}\left(\lambda\left(v_{\max } / v_{\min }-1\right) t\right)^{n}}{n !}\right] \\
& \quad= \begin{cases}0 ; & v_{\text {max }}<\frac{x}{t} \\
1-\frac{e^{\sigma_{3}}\left(v_{\max }-(x / t)\right)}{e^{\lambda\left(v_{\max } / v_{\min }-1\right) t}\left(v_{\max }-v_{\min }\right)} & v_{\min } \leq \frac{x}{t} \leq v_{\max } \\
1 ; & v_{\min }>\frac{x}{t},\end{cases}
\end{aligned}
$$

where

$$
\sigma_{3}=\frac{\lambda\left(t v_{\max }^{2}-2 t v_{\max } v_{\min }+v_{\min } x+v_{\min } x \ln \left(v_{\min }\right)-v_{\min } x \ln (x / t)\right)}{v_{\min }\left(v_{\max }-v_{\min }\right)}
$$

3.3. Derivation of PDF for $f_{L_{U C}}$ : The Distribution of Disconnected Vehicles Gap. Let $L$ denote the gap between two neighboring vehicles and let $L_{U C}$ denote the gap between two neighboring disconnected vehicles. According to Wisitpongphan et al. in [10], the spacing between two vehicles can be expressed by an exponential distribution and the validity of assumption for VANETs has been confirmed by the empirical measurement reported in [10]. Therefore, the gap between two neighboring vehicles is denoted as

$$
f_{L}(l)=\lambda_{s} e^{-\lambda_{s} l}
$$

where the parameter $\lambda_{s}$, which is the vehicle density, can be estimated as $\lambda_{s}=\lambda / E[V]$.

Two neighboring vehicles are considered disconnected if the gap between the vehicles is larger than communication range, $r$. If the spacing between two neighboring vehicles $L$ follows an exponential distribution, then the derivation of the PDF of $f_{L_{U C}}(l)$ is as follows:

$$
\begin{aligned}
& f_{L_{U C}}(l)=f_{L}(l \mid L>r), \\
& f_{L_{U C}}(l)=\lambda_{s} e^{-\lambda_{s} l} e^{\lambda_{s} r} .
\end{aligned}
$$

3.4. Derivation of $C D F$ and $P D F$ of $T_{C}$ via Numerical Integration and Approximation of $T_{C}$ Distribution. It may be noted that the CDF of $T_{C}$ given in (1) does not have a closed form solution but has to be evaluated via numerical integration. Plots in Figure 2 present the probability of $T_{C}$ distribution against time in seconds for different values of flow rate.

In general, Figure 2 indicates, with the increase in vehicle flow rates, the distance between vehicles decreases and therefore, decreases the catch-up delay. The $x$-axis in Figure 2 denotes the catch-up delay between two disconnected clusters of vehicles. A large value of $t$ indicates that the data packets are carried by an informed vehicle in a catch-up phase 


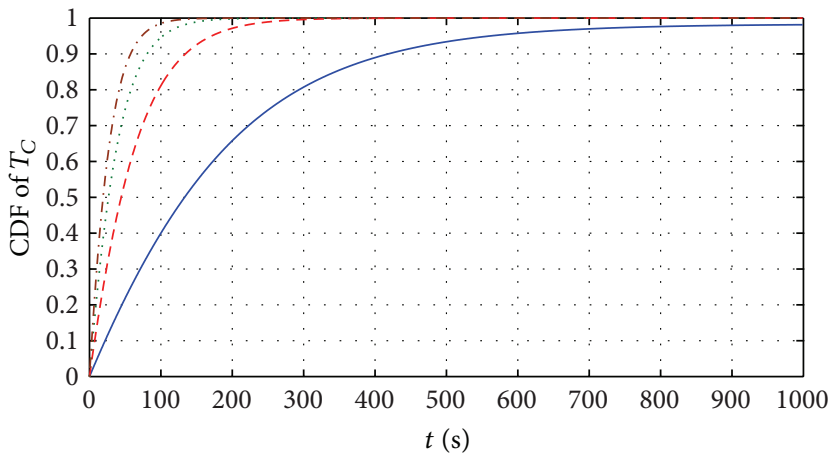

$\begin{aligned}-\lambda & =360 \mathrm{veh} / \mathrm{h} & \ldots \ldots \lambda & =1800 \mathrm{veh} / \mathrm{h} \\ --\lambda & =1080 \mathrm{veh} / \mathrm{h} & \cdots-\lambda & =2520 \mathrm{veh} / \mathrm{h}\end{aligned}$

(a) $\mathrm{CDF}$ of $T_{C}$

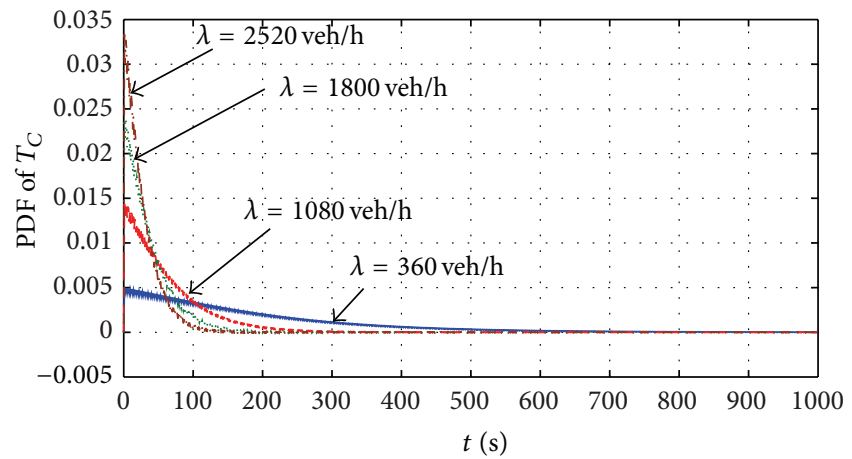

(b) PDF of $T_{\mathrm{C}}$

FIGURE 2: $F_{T_{C}}$ distribution with different values of flow rates (vehicle/hour).

most of the time and a small value of $t$ indicates that the catch-up phase occurs in a short time and the data packets are transmitted using forwarding phase most of the time. As shown in Figure 2(a), in a sparse network, the catchup phase occurs at a high delay. At $\lambda=360 \mathrm{veh} / \mathrm{hr}$, the frontmost vehicle of an informed cluster of vehicles has a probability of $70 \%$ to catch up with the last vehicle in an uninformed cluster of vehicles at a time delay $t$ larger than or equal to 200 seconds. However, in a dense network with $\lambda=2520 \mathrm{veh} / \mathrm{hr}$, the catch-up process is highly likely to happen at approximately 100 seconds or less.

Figure 2 shows that the $T_{C}$ distribution has the shape of an exponential distribution. Therefore, we use exponential regression analysis to approximate the $T_{C}$ distribution with an exponential distribution using the following equation $[17,18]$ :

$$
g(x)=a e^{b x}
$$

where $a$ and $b$ are constant called the model regression coefficients.

An exponential regression analysis is performed by applying the logarithm to the base of $e$ of both sides of (22). Subsequently, (22) can be written by

$$
\begin{gathered}
\log _{e} g(x)=\log _{e}\left(a e^{b x}\right), \\
\ln g(x)=\ln a+b x .
\end{gathered}
$$

By substituting $y=\ln g(x)$, a linear regression analysis equation, which is expressed as $y=a+b x$, can be rewritten as

$$
y=\ln a+b x,
$$

where the regression coefficients $a$ and $b$ are expressed as

$$
b=\frac{\sum x y-n \bar{x} \bar{y}}{\sum x^{2}-n \bar{x}^{2}}
$$

$\ln a=\bar{y}-b \bar{x}$,
TABLE 1: Exponential regression parameters.

\begin{tabular}{lcccc}
\hline $\begin{array}{l}\text { Vehicle } \\
\text { flow rate }\end{array}$ & SSE & $R^{2}$ & Coefficient $a$ & Coefficient $b$ \\
\hline 360 & 0.0000399 & 0.9760 & 0.005067 & -0.00498 \\
1080 & 0.0002648 & 0.9619 & 0.01465 & -0.01414 \\
1800 & 0.0006772 & 0.9448 & 0.02371 & -0.02283 \\
3600 & 0.0024381 & 0.9045 & 0.04433 & -0.04252 \\
\hline
\end{tabular}

where

$$
\begin{aligned}
\bar{x} & =\frac{1}{n} \sum_{i=1}^{n} x_{i}, \\
\bar{y} & =\frac{1}{n} \sum_{i=1}^{n} y_{i}, \\
\sum x y & =\sum_{i=1}^{n} x_{i} y_{i}, \\
\sum x^{2} & =\sum_{i=1}^{n} x_{i}^{2} .
\end{aligned}
$$

There are two main parameters in regression analysis that can indicate that an exponential distribution is a good fit for $T_{C}$ distribution $[17,18]$.

(1) Sum of Square Errors (SSE). In general, this parameter measures the difference between data points and an estimation model with a value closer to zero to indicate a good fit.

(2) Coefficient of Determination $\left(R^{2}\right)$. This parameter indicates how well data points fit an approximation curve with a value approaching one to demonstrate a good fit.

Table 1 shows the output of the exponential regression analysis for $T_{C}$ distributions for vehicle flow rates 360,1080 , 1800 , and $3600 \mathrm{veh} / \mathrm{hr}$. For each of the traffic flow rates, the exponential regression yields a high value for $R^{2}$ parameter, which is approaching one. Table 1 also indicates that the 


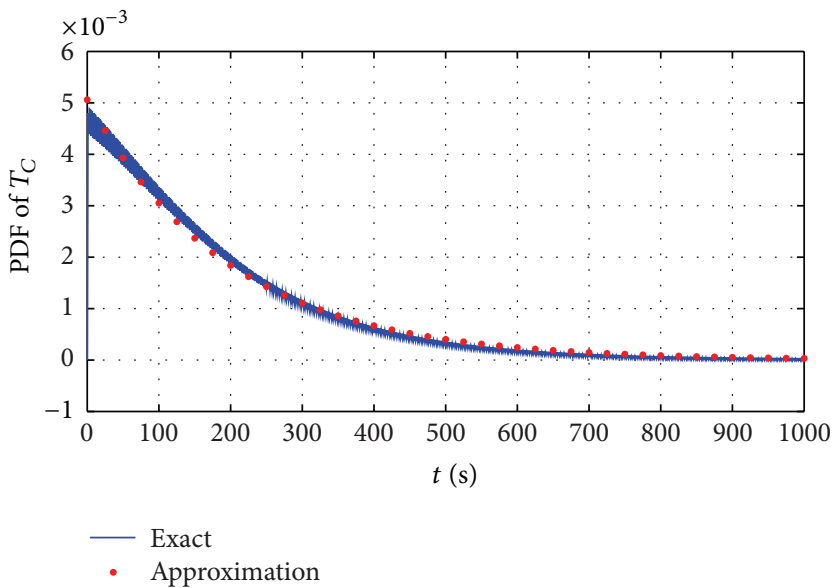

(a) $\lambda=360 \mathrm{veh} / \mathrm{hr}$

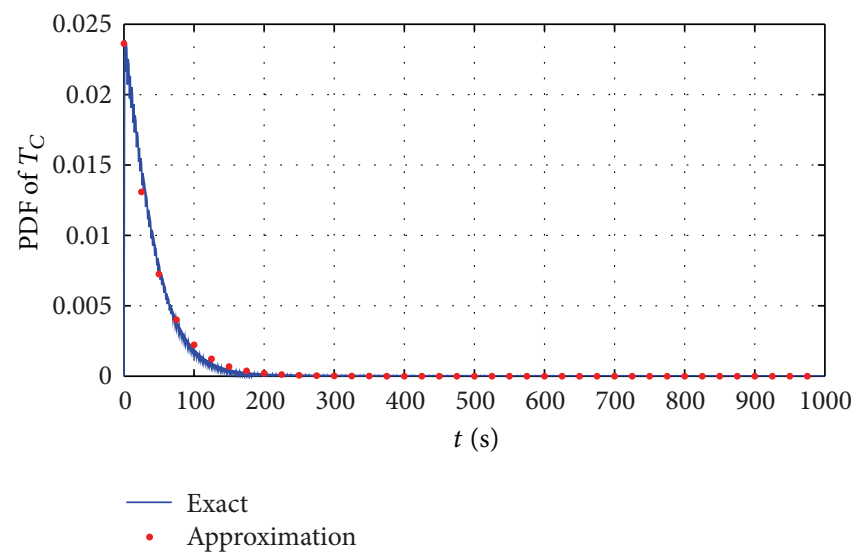

(c) $\lambda=1800 \mathrm{veh} / \mathrm{hr}$

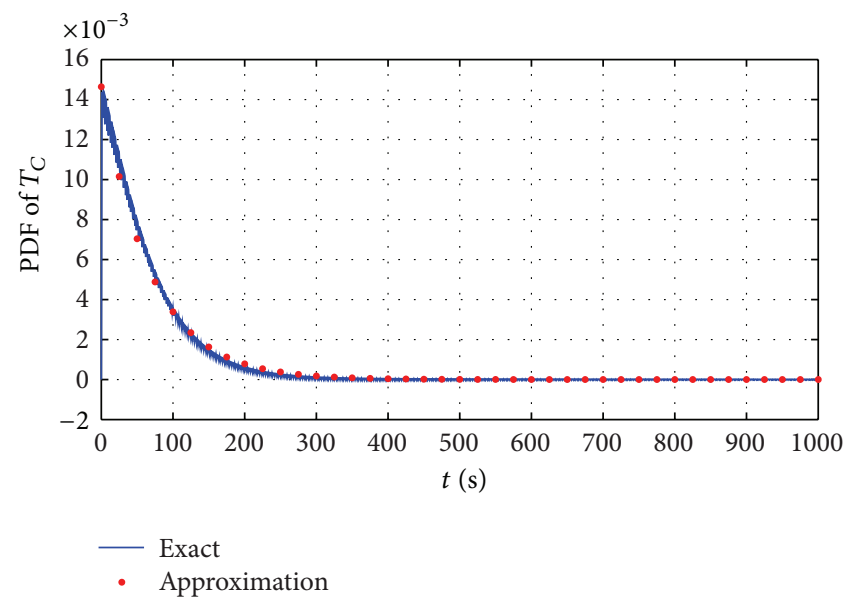

(b) $\lambda=1080 \mathrm{veh} / \mathrm{hr}$

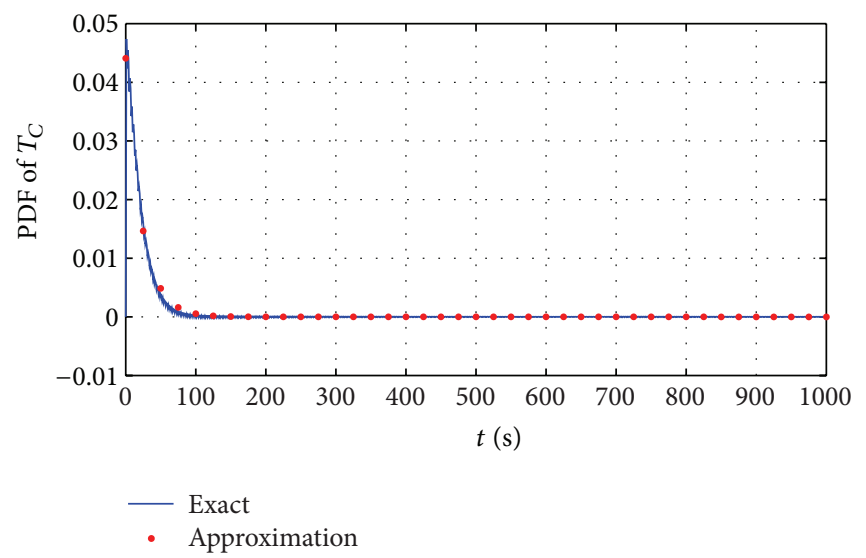

(d) $\lambda=3600 \mathrm{veh} / \mathrm{hr}$

FIgURE 3: The comparison between the exact distribution of $T_{C}$ and its approximation.

parameter SSE yields values that are very close to zero. With the parameter $R^{2}$ yielding values close to one and the SSE values producing values near to zero in the exponential regression analysis, we can ascertain that our $T_{C}$ distribution can be approximated with an exponential distribution.

Figure 3 displays the exact distribution function of $T_{C}$ with its approximation counterpart, given in (22) for the respective vehicle flow rate. From these figures, we can establish the high accuracy between the exact distribution and its approximation, and we determine that the exact distribution function of $T_{C}$ can be approximated using an exponential distribution expression.

\section{Derivation of the End-to-End Time Delay for Unidirectional Highway}

Using Figure 1 as an example, total end-to-end delay from a source vehicle to a destination vehicle, $T_{D}$, can be expressed as

$$
\begin{aligned}
T_{D}= & T_{f_{1}}+T_{c_{1}}+T_{f_{2}}+T_{\mathcal{c}_{2}}+T_{f_{3}}+T_{\mathcal{c}_{3}}+\cdots+T_{f_{n}} \\
& +T_{c_{n}},
\end{aligned}
$$

where $T_{f_{k}}$ is forwarding time in cluster $k$ and $T_{c_{k}}$ is catching up time from cluster $k$ to cluster $k+1$.
It is assumed that low vehicle density in a vehicular network causes the communication range to become smaller than the average intervehicle gap. Therefore, we ascertain that the message transmission time can be approximated using entirely the vehicle movement while ignoring the message transmission time within a cluster, that is, $T_{f_{1}}$, as it is very small, since the packets are transferred via wireless channel within the cluster, resulting in $T_{f_{1}} \ll T_{C_{1}}$. We consider that this assumption is plausible based on the current researches on performance analysis on IEEE 802.11p technology [19-21]. These researches show that the average transmission delay between two neighboring vehicles via wireless channel takes on the values of milliseconds as opposed to the transmission delay via carry and forward strategy, which has been discussed in Section 3.4.

Therefore, from the above assumption, (27) can be rewritten as

$$
T_{D}=T_{c_{1}}+T_{c_{2}}+T_{c_{3}}+\cdots+T_{c_{n}} .
$$

Generalizing (28) for $N_{c}$ clusters, we are able to derive $T_{D}$ as

$$
T_{D}=\sum_{k=1}^{N_{c}} T_{c_{k}}
$$


Let $N_{c}$ denote the number of vehicle clusters on a highway with the assumption that $N_{c}$ is a random variable that is independent of $T_{C}$ 's where $N_{c}$ derivation will be explained later in Section 4.1. Hence, we can find the conditional PDF of $T_{D}$ given that $N_{c}=n$ using the conditional characteristic function of $T_{D}$. From (28), the conditional characteristic function of $T_{D}$ given that $N_{c}=n$ can be expressed as

$$
\begin{aligned}
T_{D} & =T_{C_{1}}+T_{C_{2}}+\cdots+T_{C_{n}}, \\
\Phi_{T_{D} \mid N_{c}=n}(s) & =E\left[e^{s T_{D}}\right]=E\left[e^{s\left(T_{C_{1}}+T_{C_{2}}+\cdots+T_{C_{N}}\right)}\right], \\
\Phi_{T_{D} \mid N_{c}=n}(s) & =\left\{\Phi_{T_{C}}(s)\right\}^{n} .
\end{aligned}
$$

The conditional PDF of $T_{D}$ can be found by taking the inverse transform of $\Phi_{T_{D} \mid N_{c}=n}$. However, since the distribution of $T_{C}$ in (1) is found using numerical integration, a closed form solution is not feasible for (30). In Section 3.4, using exponential regression analysis, we have determined that the catch-up time $\left(T_{C}\right)$ distribution in Figure 2 can be approximated with an exponential distribution. Henceforth, $f_{T_{\mathrm{C}}}$ can be denoted with the following expression:

$$
f_{T_{C}}(t) \approx \lambda_{T_{C}} e^{-\lambda_{T_{C}} t}
$$

where the value of $\lambda_{T_{C}}$ should be found using minimum mean square error (MMSE) between the exact distribution function of $T_{C}$ in (1) and its approximation. Using the approximation of $T_{C}$ as an exponential distribution, the conditional PDF of $T_{D}$ given that $N_{c}=n$ can be found using characteristic function expressions of $T_{C}$ and sums of $T_{C}$ 's. The characteristic function of $T_{C}$ can be expressed as

$$
\begin{aligned}
\Phi_{T_{C}}(s) & =E\left[e^{s T_{C}}\right]=\int_{0}^{\infty} e^{s t} f_{T_{C}} d t \\
& =\int_{0}^{\infty} e^{s t} \lambda_{T_{C}} e^{-\lambda_{T_{C}} t} d t, \\
\Phi_{T_{C}}(s) & =\frac{\lambda_{T_{C}}}{\left(\lambda_{T_{C}}-s\right)} .
\end{aligned}
$$

From (32), we can find the expression for the characteristic function of $T_{D}$ given that $N_{C}=n$ :

$$
\begin{aligned}
T_{D} & =T_{C_{1}}+T_{C_{2}}+\cdots+T_{C_{n}}, \\
\Phi_{T_{D} \mid N_{c}=n}(s) & =E\left[e^{s T_{D}}\right]=E\left[e^{s\left(T_{C_{1}}+T_{C_{2}}+\cdots+T_{C_{n}}\right)}\right] \\
& =\Phi_{T_{C_{1}}}(s) \cdots \Phi_{T_{C_{N}}}(s), \\
\Phi_{T_{D} \mid N_{c}=n}(s) & =\left\{\Phi_{T_{C}}(s)\right\}^{n}, \\
\Phi_{T_{D} \mid N_{c}=n}(s) & =\left\{\frac{\lambda_{T_{C}}}{\left(\lambda_{T_{C}}-s\right)}\right\}^{n} .
\end{aligned}
$$

Thus, the conditional PDF of $T_{D}$ can be found using inverse Laplace transform of the conditional characteristic function of $T_{D}$ in (33) which is expressed in

$$
\begin{aligned}
f_{T_{D} \mid N_{c}=n}(t) & =\mathscr{L}^{-1}\left\{\Phi_{T_{D}}(s)\right\} \\
& =\mathscr{L}^{-1}\left\{\left\{\frac{\lambda_{T_{C}}}{\left(\lambda_{T_{C}}-s\right)}\right\}^{n}\right\}, \\
f_{T_{D} \mid N_{c}=n}(t) & =\frac{\lambda_{T_{C}} e^{-\lambda_{T_{C}} t}\left(\lambda_{T_{C}} t\right)^{n-1}}{(n-1) !} .
\end{aligned}
$$

From (34), we ascertain that the conditional PDF of $T_{D}$ given that $N_{C}=n$ follows an $\operatorname{Erlang}\left(n, \lambda_{T_{C}}\right)$ distribution.

4.1. Analysis on the Distribution of Number of Clusters, $N_{C}$. To find the PDF of $T_{D}$, we have to derive the distribution model for the number of clusters, $N_{C}$. Based on the message propagation scenario in Figure 1 of Section 3, we consider a unidirectional highway of length $D$ meters. The source vehicle is located in the first informed cluster and the destination vehicle is located at the end of the highway. Therefore, a message from the source vehicle has to be propagated over multiple clusters of vehicles in order to be transmitted to the destination vehicle. In addition, we assume that the vehicles enter the highway according to a Poisson process with traffic flow rate of $\lambda$. Therefore, by employing the Poisson process assumption, we consider that the number of clusters can be modeled by using the Poisson distribution. Subsequently, by applying the vehicular network analytical framework provided in [3], we are able to formulate a distribution model for number of clusters for a unidirectional highway of length D.

Let $N_{C}$ denote number of clusters in a unidirectional highway and its derivation is based on vehicular network parameters provided in [3].

4.1.1. Average Distance during Forwarding Phase, $E\left[X_{f}\right]$. Let $X_{f}$ represent the distance traveled by messages during a forwarding phase where the expected value is given as

$$
E\left[X_{f}\right]=E[Y]+r,
$$

where $r$ is the transmission range and $E[Y]$ is the average cluster size and it is expressed as

$$
E[Y]=E[M] E\left[L_{c}\right],
$$

where $E[M]$ is the average number of vehicle gaps in a cluster and $E\left[L_{c}\right]$ is the average gap between two connected neighboring vehicles.

Next, we derive the following closed form expressions for $E[M]$ and $E\left[L_{c}\right]$, respectively:

$$
\begin{aligned}
& E[M]=\frac{F_{L}(r)}{1-F_{L}(r)}=\frac{1-e^{-\lambda_{s} r}}{e^{-\lambda_{s} r}}, \\
& E\left[L_{c}\right]=\int_{0}^{r} l \frac{f_{L}(l)}{f_{L}(r)} d l=\frac{1-\left(e^{-\lambda_{s} r}\left(\lambda_{s} r+1\right)\right)}{\lambda_{s}\left(1-e^{-\lambda_{s} r}\right)} .
\end{aligned}
$$


Therefore, using (37) and (38), (35) can be solved as follows:

$$
\begin{aligned}
& E\left[X_{f}\right]=r+E[M] E\left[L_{c}\right], \\
& E\left[X_{f}\right]=r+\frac{e^{-\lambda_{s} r}\left(1-\left(e^{-\lambda_{s} r}\left(\lambda_{s} r+1\right)\right)\right)}{\lambda_{s}} .
\end{aligned}
$$

4.1.2. Average Distance during Catch-Up Phase, $E\left[X_{c}\right]$. Let $X_{c}$ denote distance traveled by messages during a catch-up phase and the expected value is expressed as

$$
\begin{aligned}
E\left[X_{c}\right] & =\int_{0}^{\infty} E\left[X_{c} \mid T_{c}=t\right] f_{T_{C}}(t) d t \\
& =\int_{0}^{\infty} E[X(t)] f_{T_{C}}(t) d t .
\end{aligned}
$$

It may be noted that the closed form expression is not feasible for (40) since $E[X(t)]$ is solved using numerical integration. Therefore, (40) is solved through numerical integration using the exact distribution of $T_{C}$ in (1).

Finally, the probability mass function for $N_{C}$ can be expressed as

$$
P(N=n)=\frac{\alpha^{n}}{n !} e^{\alpha}, \quad \text { with } \alpha=\frac{D}{E\left[X_{f}\right]+E\left[X_{C}\right]},
$$

where $D$ is the length of the highway, $E\left[X_{f}\right]$ is the expected value of the forwarding distance, and $E\left[X_{C}\right]$ is the expected value of the catch-up distance.

4.2. Derivation of $T_{D}$ Distribution Based on $T_{C}$ and $N_{c}$ Distributions. It is stated in $[15,16]$ that the Erlang distribution is obtained by the $n$-fold convolution of $n$ independent exponential distribution. In Section 4, we have shown that the distribution of $T_{D}$ is the summation of the catch-up delay, $T_{C}$, for $n$ vehicle clusters. In addition, we establish that $T_{C}$ is a random variable that exhibits the properties of an exponential distribution.

Therefore, let $T_{D}$ denote the sum of the $T_{C}$ for $N_{C}=n$ and let $N_{C}$ denote the number of vehicle clusters on a unidirectional highway. Based on the Law of Total Probability, the PDF of $T_{D}$ can be formulated as

$$
\begin{aligned}
f\left(T_{D} ; t\right)= & f_{T_{D}}\left(t \mid N_{c}=n_{1}\right) P\left(N_{c}=n_{1}\right) \\
& +f_{T_{D}}\left(t \mid N_{c}=n_{2}\right) P\left(N_{c}=n_{2}\right) \\
& +f_{T_{D}}\left(t \mid N_{c}=n_{3}\right) P\left(N_{c}=n_{3}\right) \cdots, \\
f\left(T_{D} ; t\right)= & \sum_{n=1}^{\infty} \frac{\lambda_{T_{C}} e^{-\lambda_{T_{C}} t}\left(\lambda_{T_{C}} t\right)^{n-1}}{(n-1) !} \cdot \frac{\alpha_{i}^{n}}{n !} e^{\alpha},
\end{aligned}
$$

where

$$
\alpha=\frac{D}{E\left[X_{f}\right]+E\left[X_{C}\right]} .
$$

The PDF of $T_{D}$, as shown in (42), exhibits properties of an Erlang distribution, which will be proved in Section 5.1.

\section{Results and Analysis for Distribution of Total Catch-Up Time $T_{D}$ in One-Way Street}

5.1. Numerical Results. In this section, we present some pertinent numerical results regarding the analysis done in this chapter. Figure 4 shows the probability mass functions for $N_{C}$ from (41) and for traffic flow rate $\lambda=360,1080,1800$ and $2520 \mathrm{veh} / \mathrm{hr}$. It can be seen in Figure 4 that, at $\lambda=$ $360 \mathrm{veh} / \mathrm{hr}$, the peak of the distributions occurs at $n=6$ and at $\lambda=2520 \mathrm{veh} / \mathrm{hr}$; the peak of the distribution is at $n=1$. Consequently, the plots in Figure 4 demonstrate that as the traffic density in a highway increases, clusters in the highway merge to form a larger cluster, thereby reducing number of clusters in the highway.

In addition, there is a correlation between number of clusters and traffic density where the number of clusters decreases as traffic density increases. The relationship is shown in Figure 5, which presents the average distance traveled by messages during forward phase, $E\left[X_{f}\right]$, and catch-up phase, $E\left[X_{c}\right]$. Figure 5 shows that the value of $E\left[X_{f}\right]$ also increases with the increment of traffic flow rate, confirming that as the traffic density increases, messages are mostly transmitted via wireless channel rather than being carried by vehicles. From Figure 5, we ascertain that the average cluster length increases as vehicle traffic flow rate increases; the intervehicle spacing reduces until the gap is less than the transmission range.

Furthermore, the trend in the plots of $E\left[X_{f}\right]$ and $E\left[X_{c}\right]$ in Figure 5 derived from our analysis in Section 4.1 shows an exact match with the numerical results of the original $E\left[X_{f}\right]$ and $E\left[X_{c}\right]$ from [3]. The same trend displayed in Figure 5 and the original result in [3] validated the accuracy of our work on the distribution of $T_{C}$ from [3]. Figure 6 displayed the information propagation speed based on the $T_{D}$ distribution, which further validates the accuracy of our analysis as Figure 6 shows similar trend with the information propagation speed from [3]. Therefore, we are able to arrive with the same conclusion as [3] in which higher vehicle density leads to a larger partition size and shorter intercluster distance and henceforth reduces the catch-up time. Figure 6 shows a sharp increase as the vehicle flow rate increases and the propagation speed is shown much faster than the vehicle movement.

Figure 7 displays the PDF of $T_{D}$ distribution. The probability of $T_{D}$ is plotted against the time delay in seconds for different vehicles flow rates. As shown in Figure 7, the PDF of $T_{D}$ exhibits similarities to an Erlang - $n$ distribution, which confirms our analysis that the PDF of $T_{D}$ follows Erlang $-n$ distribution given that $N_{C}=n$.

5.2. Simulation Results. In this section, we present simulation results in Figures 9(a)-9(c) for our proposed analytical model using the network simulator NS-2 [22, 23]. Our simulation scenario, which is displayed in Figure 8, is based on a onedirectional highway with the length of $15 \mathrm{~km}$. The highway is assumed to have multiple one-directional lanes, where vehicles can overtake each other without changing their lane or maneuvering. Vehicles are generated using a Poisson process with flow rates of $360,1080,1800$, and $3600 \mathrm{veh} /$ hour. 


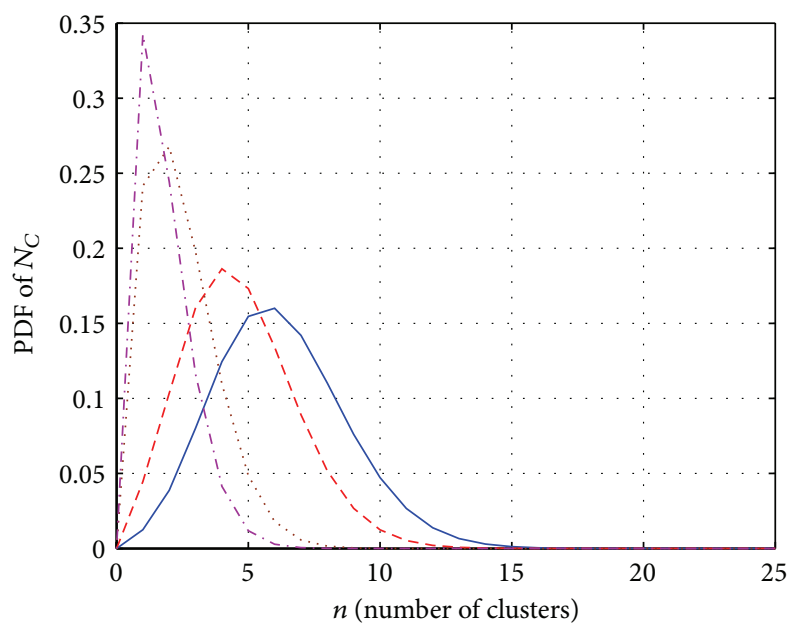

$$
\begin{aligned}
-\lambda & =360 \mathrm{veh} / \mathrm{h} & \cdots \cdots \lambda & =1800 \mathrm{veh} / \mathrm{h} \\
--\lambda & =1080 \mathrm{veh} / \mathrm{h} & \cdots-\lambda & =2520 \mathrm{veh} / \mathrm{h}
\end{aligned}
$$

FIGURE 4: PDF of $N_{C}$.

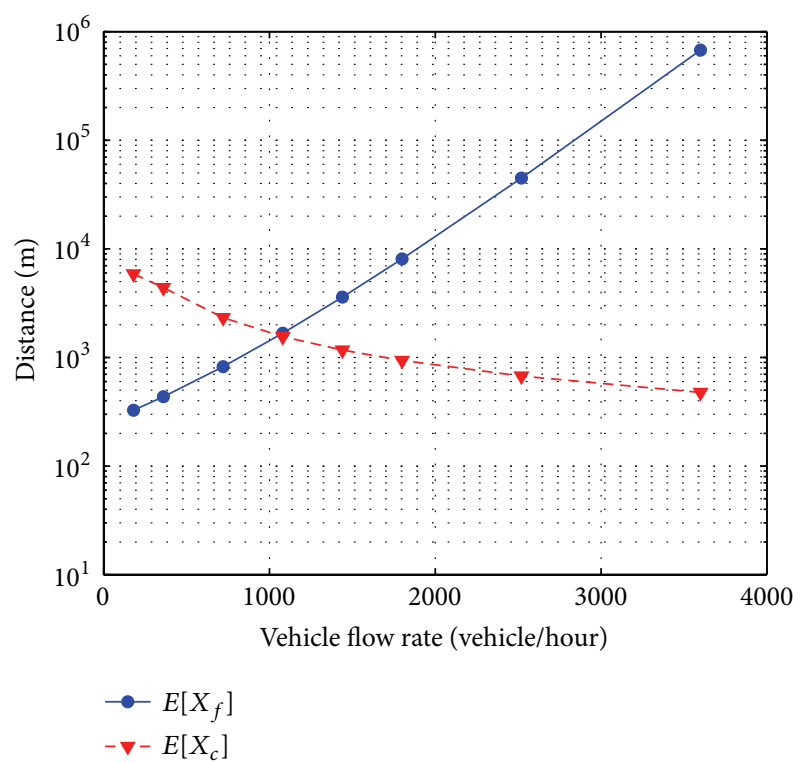

Figure 5: Expected values of $X_{f}$ and $X_{c}$ for $N_{C}$ analysis.

Each vehicle is assigned a random speed based on a uniform distribution between the intervals $v_{\min }=20 \mathrm{~m} / \mathrm{s}$ and $v_{\max }=$ $28.89 \mathrm{~m} / \mathrm{s}$ and the assigned speed does not change over the simulation time. We perform the simulation for 1200 seconds and repeat the simulation for 1000 iterations. Since NS-2 is built to simulate a network environment, we configure media access control (MAC) and physical (PHY) layers in NS-2 to retain [3] assumptions of ideal MAC and PHY layers for the model so that the simulation is executed under ideal communication channel. The packets are generated using Poisson traffic with mean of 0.1 seconds and the transmission range is set to 250 meters. In addition, we configure the source vehicle to be the only informed vehicle at time $t$ and to be located at position $H$; and the destination vehicle is the first vehicle to pass location $H$ at time $t$.

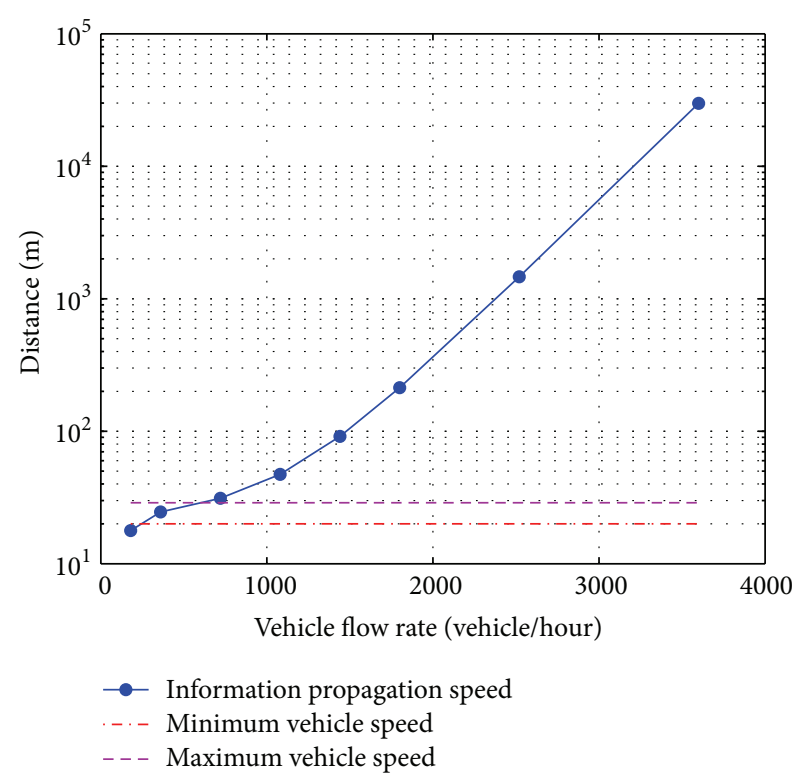

FIgURE 6: Information propagation speed from $T_{D}$ analysis.

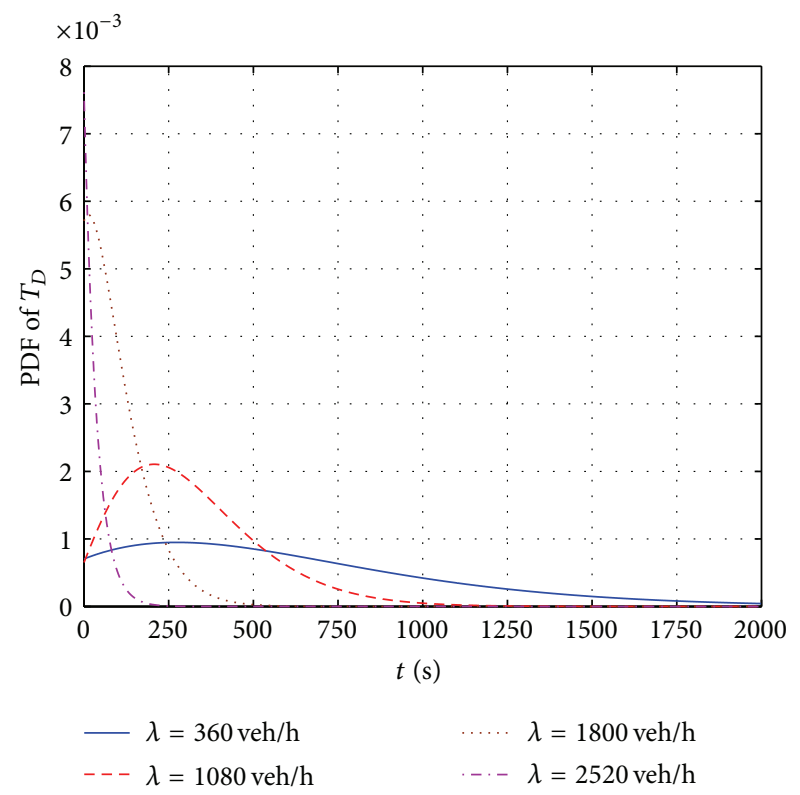

FIgURE 7: The PDF of $T_{D}$.

Figure 9 displays the comparison between the numerical results of the $T_{D}$ analysis with the simulation results. Figure 9(a) presents the numerical and simulation results for the PDF of $T_{D}$ from (42). The results are plotted as a function of the time delay with different values of traffic flow rates. From Figure 9(a), at $\lambda=360 \mathrm{veh} / \mathrm{hr}$, the simulation result shows that the peak of the distribution occurs at approximately $t=$ 300 seconds, whereas, at $\lambda=1800 \mathrm{veh} / \mathrm{hr}$, the distribution's peak is at $t=50$ seconds. It can be concluded that as the traffic flow rate increases, the end-to-end delay decreases since the traffic density on the highway will be large enough to allow packet transmission via multihop forwarding on the wireless channel. 


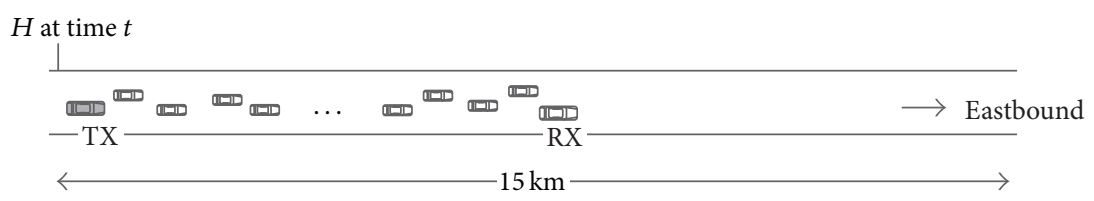

Figure 8: Simulation scenario.

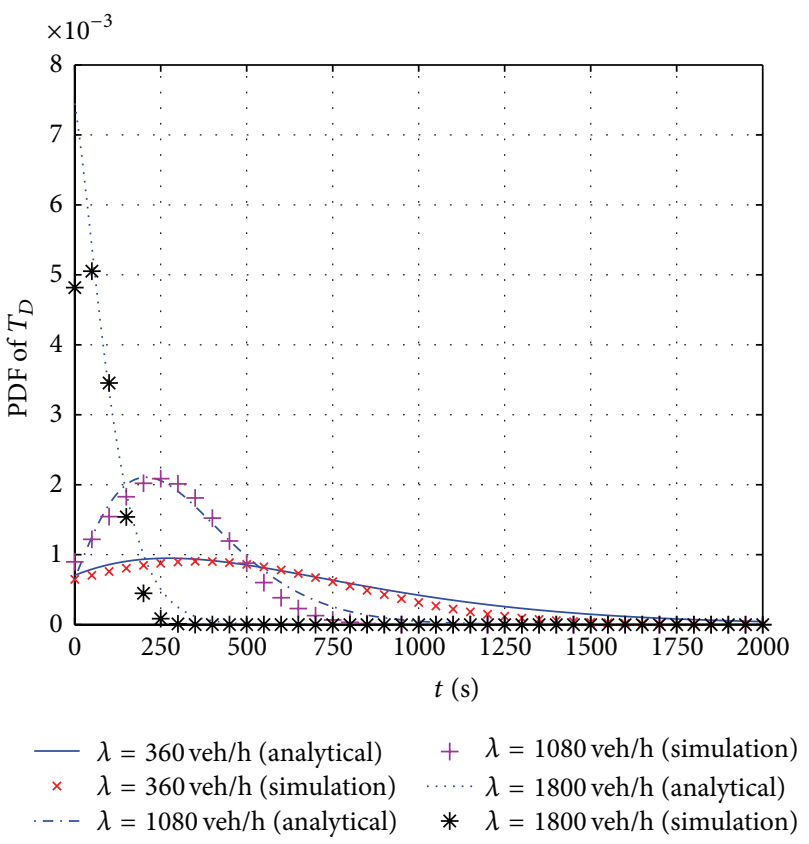

(a) The PDF of $T_{D}$ with different values of traffic flow rates

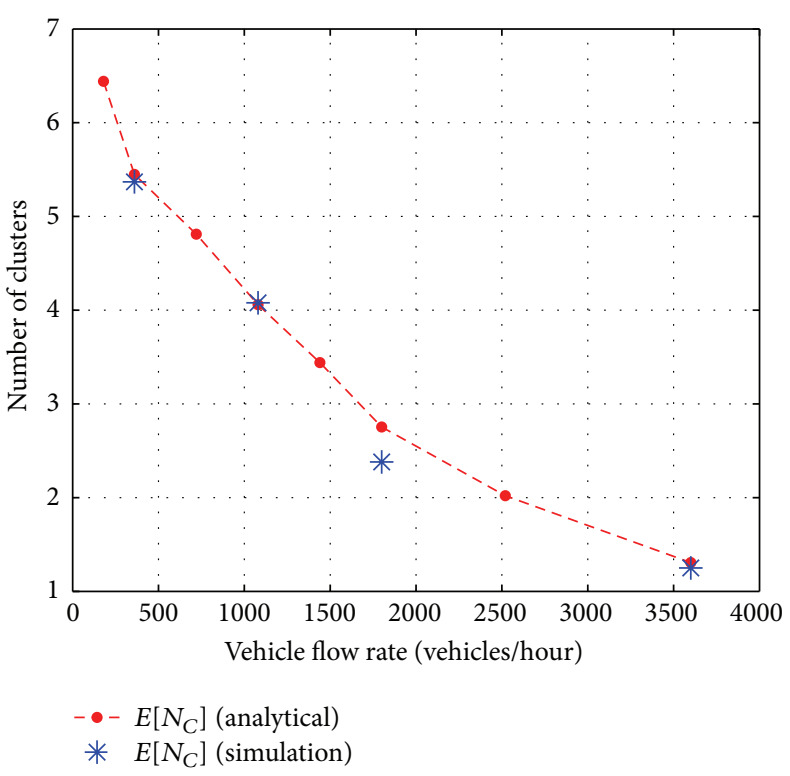

(b) Expected number of clusters

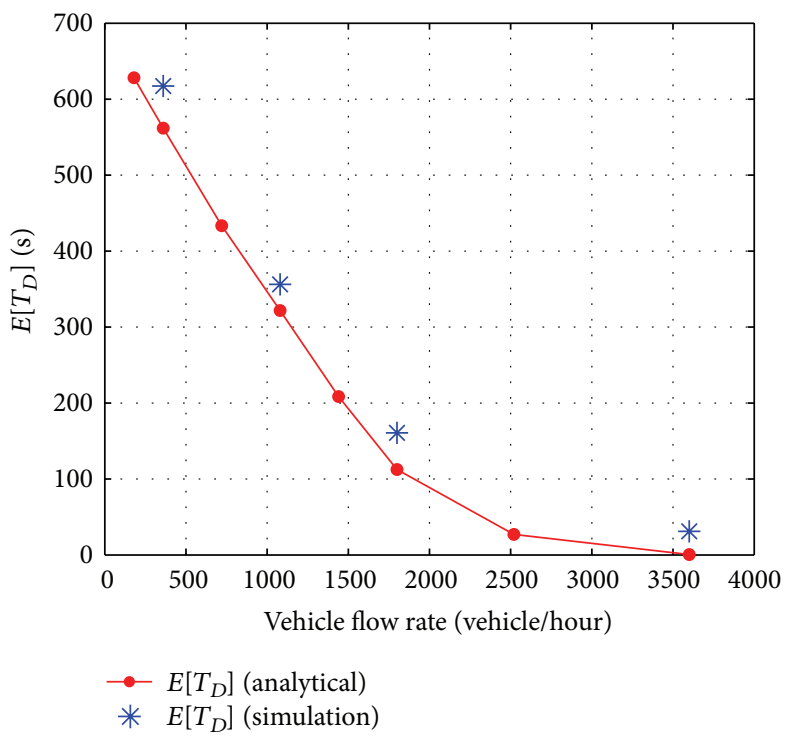

(c) Expected end-to-end delay

FIGURE 9: Comparison between analytical and simulation results of $T_{D}$ distribution model.

In addition, the plots for $T_{D}$ distribution in Figure 9(a) show that the numerical and simulation plots of $T_{D}$ are very close to each other and exhibit the same trend as the Erlang $-n$ distribution model. From Figure 9, we ascertain that the small discrepancy between the analytical plots of $T_{D}$ distribution and the plots of $T_{D}$ simulation is caused by the configuration of NS-2. We conclude that even though NS-2 has been configured with the ideal communication channel, MAC and PHY conditions in NS-2 still affect the simulation results. Furthermore, we ascertain that the use of Poisson 
traffic for data packets generation in the simulation causes a small deviation between analytical and simulation results.

\section{Conclusion}

In this section, we propose an analytical framework for the end-to-end delay model for a vehicular network on a unidirectional highway by extending the catch-up delay model between two adjacent vehicle clusters to multiple vehicle clusters as well as using traffic characteristics models to determine the distribution model for number of clusters. We approximate the distribution of the catch-up delay model between two disconnected clusters using the exponential regression analysis to derive the catch-up delay model for multiple clusters. Using the approximation, we establish that the catch-up delay model for multiple clusters follows an Erlang $-n$ distribution. We also validated our analytical results through simulation. In this study, we are able to confirm that the carry and forward process often occurs in a low density network and the process has caused the packets to be delivered at high end-to-end delay. Nonetheless, the study is done without considering a number of real-world communication aspects such as channel fading and contention issues at media access layer which can be considered as the research future works.

\section{Notations}

$$
\begin{aligned}
& \text { D: } \quad \text { The length of a road } \\
& \text { L: } \quad \text { Gap between two neighboring } \\
& \text { vehicles } \\
& L_{c} \text { : } \quad \text { Gap between two neighboring } \\
& \text { connected vehicles } \\
& L_{U C} \text { : Gap between two neighboring } \\
& \text { disconnected vehicles } \\
& \lambda: \quad \text { Traffic flow rate (vehicles/unit time) } \\
& \lambda_{s}: \quad \text { Vehicles density (vehicles/unit } \\
& \text { distance) } \\
& \lambda_{T_{\mathrm{C}}} \text { : Minimum mean square error } \\
& \text { between the exact distribution of } T_{C} \\
& \text { and an exponential distribution } \\
& N(t) \text { : Number of vehicles arriving at the } \\
& \text { highway during interval }[0, t] \\
& N_{c} \text { : Number of vehicle clusters }
\end{aligned}
$$

$X(t)$ : Message propagation distance during $(0, t]$

$X^{\prime}(t)$ : Distance that the partition tail moves during $[0, t]$

$Y$ : The size of a cluster.

\section{Conflict of Interests}

The authors declare that there is no conflict of interests regarding the publication of this paper.

\section{Acknowledgments}

The authors would like to thank Universiti Teknikal Malaysia Melaka (UTeM) and Memorial University of Newfoundland for their continuous financial support in making this research possible.

\section{References}

[1] G. Karagiannis, O. Altintas, E. Ekici et al., "Vehicular networking: a survey and tutorial on requirements, architectures, challenges, standards and solutions," IEEE Communications Surveys and Tutorials, vol. 13, no. 4, pp. 584-616, 2011.

[2] E. Schoch, F. Kargl, M. Weber, and T. Leinmuller, "Communication patterns in VANETs," IEEE Communications Magazine, vol. 46, no. 11, pp. 119-125, 2008.

[3] H. Wu, R. M. Fujimoto, G. F. Riley, and M. Hunter, "Spatial propagation of information in vehicular networks," IEEE Transactions on Vehicular Technology, vol. 58, no. 1, pp. 420-431, 2009.

[4] S. Durrani, X. Zhou, and A. Chandra, "Effect of vehicle mobility on connectivity of vehicular ad hoc networks," in Proceedings of the IEEE 72nd Vehicular Technology Conference Fall (VTC-Fall '10), pp. 1-5, September 2010.

[5] A. Kesting, M. Treiber, and D. Helbing, "Connectivity statistics of store-and-forward intervehicle communication," IEEE Transactions on Intelligent Transportation Systems, vol. 11, no. 1, pp. 172-181, 2010.

[6] Z. Zhang, G. Mao, and B. D. O. Anderson, "On the information propagation process in mobile vehicular ad hoc networks," IEEE Transactions on Vehicular Technology, vol. 60, no. 5, pp. 23142325, 2011.

[7] Z. Zhang, G. Mao, and B. D. O. Anderson, "Stochastic characterization of information propagation process in vehicular ad hoc networks," IEEE Transactions on Intelligent Transportation Systems, vol. 15, no. 1, pp. 122-135, 2014.

[8] A. Agarwal, D. Starobinski, and T. D. C. Little, "Phase transition of message propagation speed in delay-tolerant vehicular networks," IEEE Transactions on Intelligent Transportation Systems, vol. 13, no. 1, pp. 249-263, 2012.

[9] E. Baccelli, P. Jacquet, B. Mans, and G. Rodolakis, "Highway vehicular delay tolerant networks: information propagation speed properties," IEEE Transactions on Information Theory, vol. 58, no. 3, pp. 1743-1756, 2012.

[10] N. Wisitpongphan, F. Bai, P. Mudalige, V. Sadekar, and O. Tonguz, "Routing in sparse vehicular ad hoc wireless networks," IEEE Journal on Selected Areas in Communications, vol. 25, no. 8, pp. 1538-1556, 2007.

[11] T. A. Khalaf and S. W. Kim, "Delay analysis in message ferrying system," in Proceedings of the IEEE International Conference 
on Electro/Information Technology (EIT '08), pp. 333-336, May 2008.

[12] J. Fukuyama, "A delay time analysis for multi-hop V2V communications over a linear VANET," in Proceedings of the IEEE Vehicular Networking Conference (VNC '09), pp. 1-7, IEEE, Tokyo, Japan, October 2009.

[13] A. Abdrabou and W. Zhuang, "Probabilistic delay control and road side unit placement for vehicular ad hoc networks with disrupted connectivity," IEEE Journal on Selected Areas in Communications, vol. 29, no. 1, pp. 129-139, 2011.

[14] H. Füßler, M. Mauve, H. Hartenstein, M. Käsemann, and D. Vollmer, "MobiCom poster: location-based routing for vehicular ad-hoc networks," ACM SIGMOBILE Mobile Computing and Communications Review, vol. 7, no. 1, pp. 47-49, 2003.

[15] S. M. Ross, Introduction to Probability Models, Academic Press, Orlando, Fla, USA, 10th edition, 2009.

[16] A. Leon-Garcia, Probability and Random Processes for Electrical Engineering, Addison-Wesley, Reading, Mass, USA, 2nd edition, 1994.

[17] S. Chatterjee and B. Price, Regression Analysis by Example, Wiley-Interscience, 4th edition, 2006.

[18] R. Jain, The Art of Computer Systems Performance Analysis: Techniques for Experimental Design, Measurement, Simulation, and Modeling, Wiley Professional Computing, Wiley, 1991.

[19] M. I. Hassan, H. L. Vu, and T. Sakurai, "Performance analysis of the IEEE 802.11 MAC protocol for DSRC safety applications," IEEE Transactions on Vehicular Technology, vol. 60, no. 8, pp. 3882-3896, 2011.

[20] Y. Yao, L. Rao, and X. Liu, "Performance and reliability analysis of IEEE 802.11p safety communication in a highway environment," IEEE Transactions on Vehicular Technology, vol. 62, no. 9, pp. 4198-4212, 2013.

[21] K. A. Hafeez, L. Zhao, B. Ma, and J. W. Mark, "Performance analysis and enhancement of the DSRC for VANET's safety applications," IEEE Transactions on Vehicular Technology, vol. 62, no. 7, pp. 3069-3083, 2013.

[22] T. Issariyakul and E. Hossain, Introduction to Network Simulator NS2, vol. 1st, Springer, Berlin, Germany, 2008.

[23] The Network Simulator NS-2, http://www.isi.edu/nsnam/ns/. 


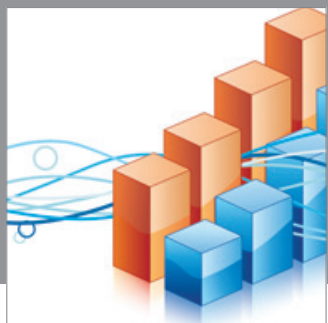

Advances in

Operations Research

mansans

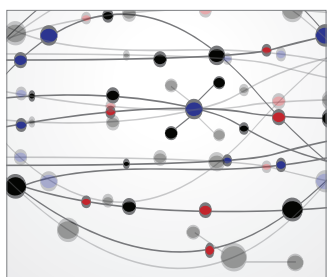

The Scientific World Journal
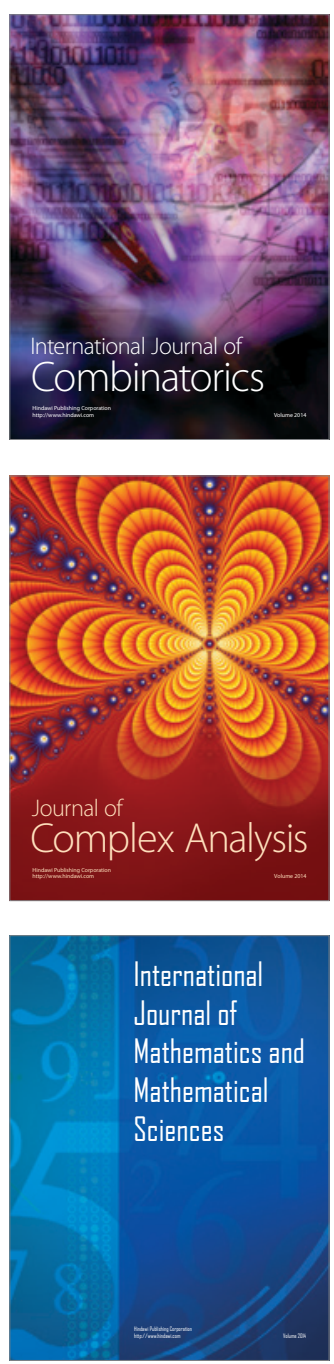
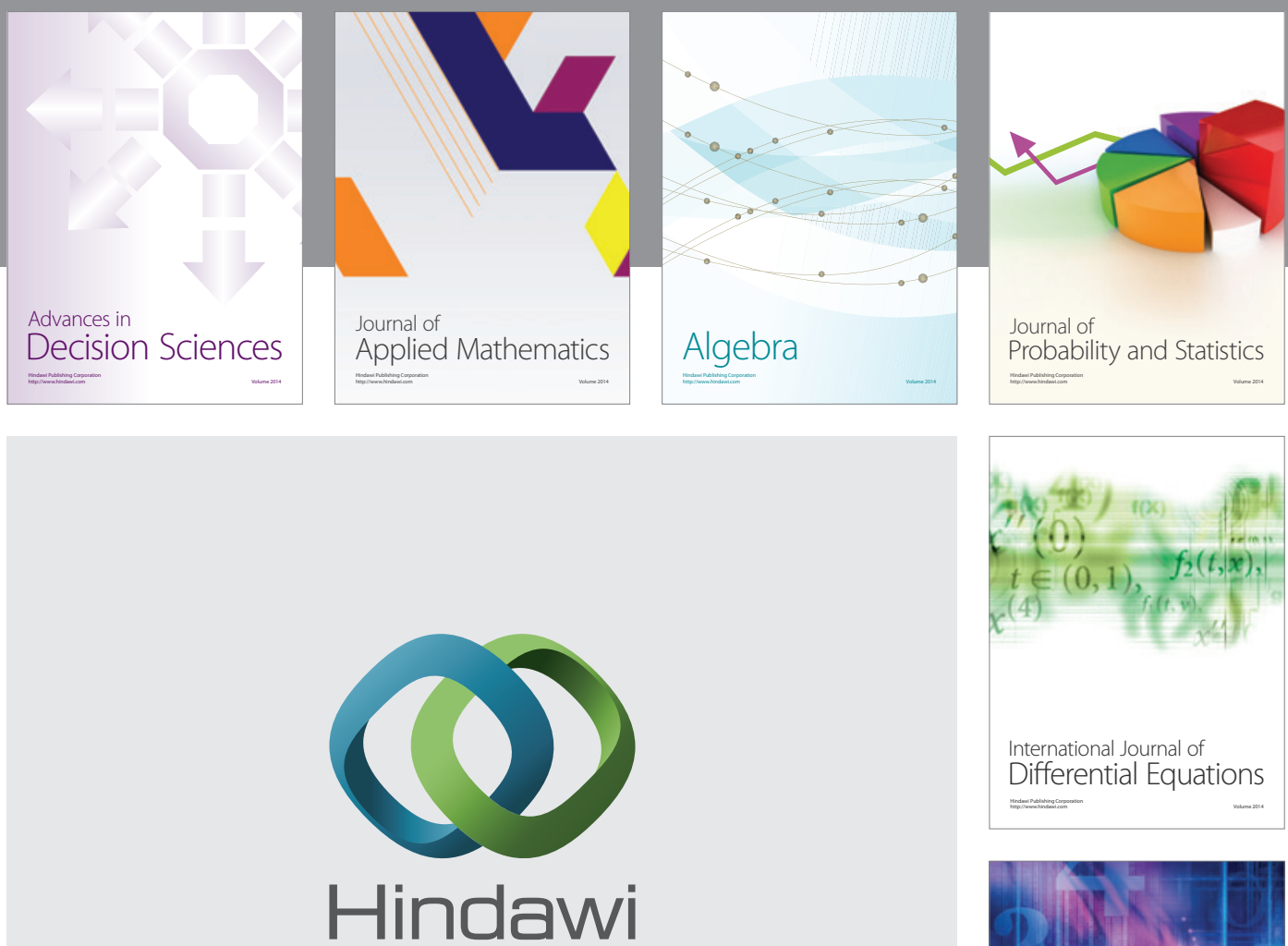

Submit your manuscripts at http://www.hindawi.com
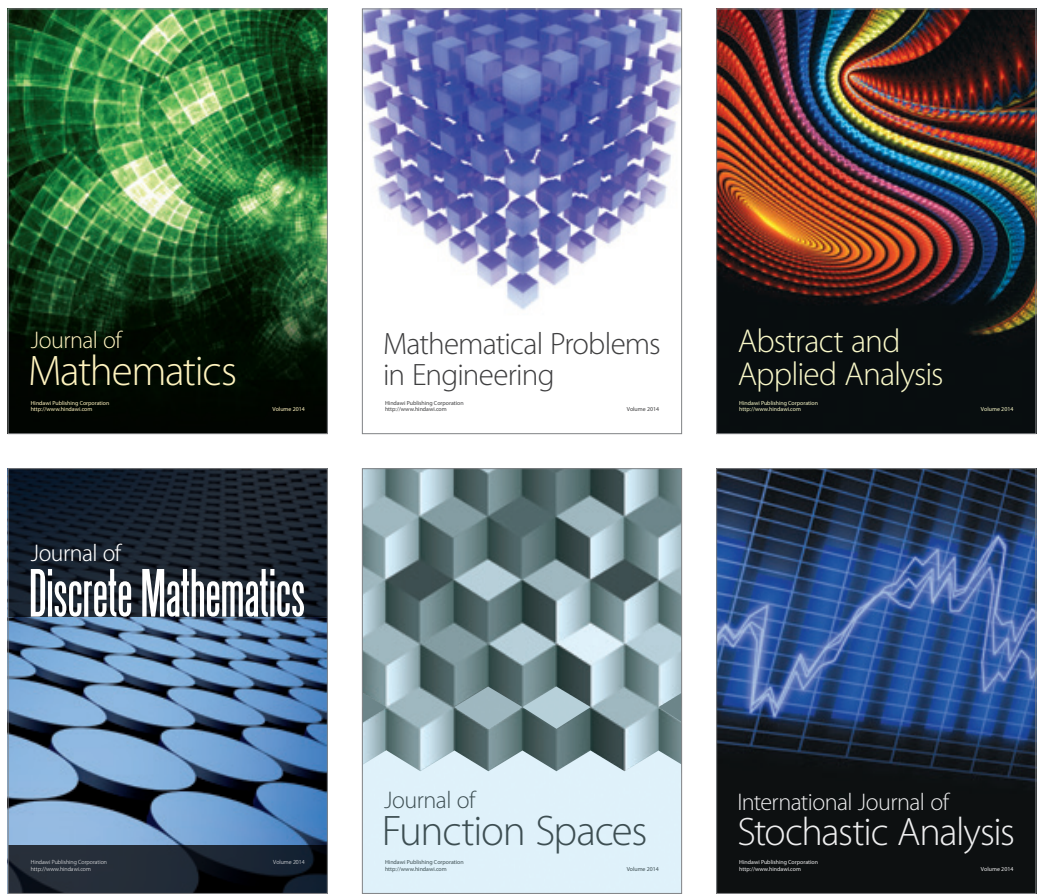

Journal of

Function Spaces

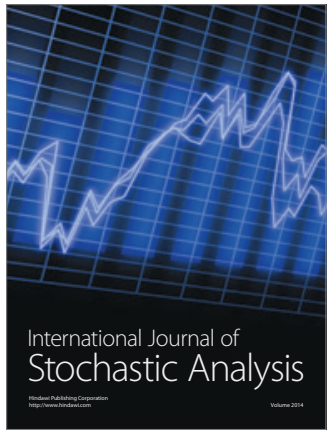

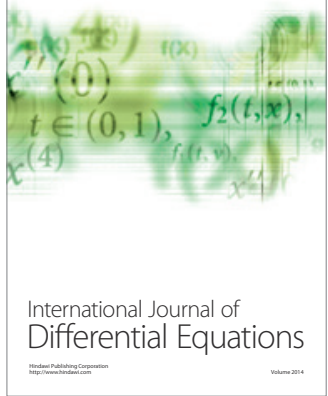
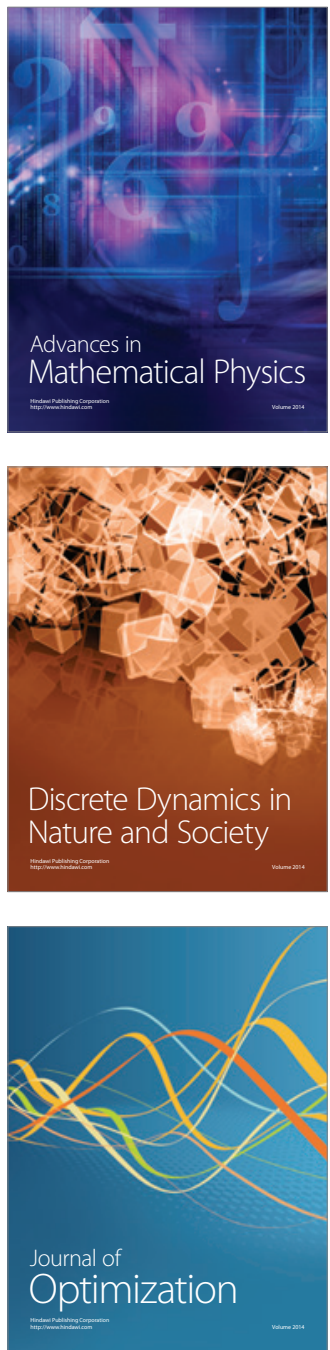